\title{
Preparation of Encapsulated Resveratrol Liposome Thermosensitive Gel and Evaluation of Its Capability to Repair Sciatic Nerve Injury in Rats
}

\author{
Hui Yao, Hao Lu, Rong Zou, Xiwen Chen, and Hanlin Xu \\ College of Pharmacy, Hubei University of Chinese Medicine, Wuhan, Hubei, China \\ Correspondence should be addressed to Hanlin Xu; xhl@hbtcm.edu.cn
}

Received 23 April 2020; Accepted 31 July 2020; Published 28 August 2020

Academic Editor: Laura Martinez Maestro

Copyright (C) 2020 Hui Yao et al. This is an open access article distributed under the Creative Commons Attribution License, which permits unrestricted use, distribution, and reproduction in any medium, provided the original work is properly cited.

\begin{abstract}
The purpose of this study was to prepare a liposomal temperature-sensitive gel able to slowly release resveratrol after local intramuscular injection. The best formulation of resveratrol liposomes was based on the highest encapsulation efficiency and drug loading designed by Box-Behnken. The prepared liposomes were approximately circular, with a mean particle diameter of $161.5 \pm 0.12 \mathrm{~nm}$ and zeta potential of $-6.9 \mathrm{mV}$. The optimized liposomes were dispersed in a polymer gel (PLGA-PEG-PLGA) for preparation of an in situ-formed gel at $35 \pm 2^{\circ} \mathrm{C}$. In vitro release of the prepared liposome temperature-sensitive gel was studied and compared with ordinary drug-releasing gels, revealing a significantly longer drug release time. Finally, a rat sciatic nerve injury model was used to evaluate the pharmacological activity of the liposome temperature-sensitive gels for the repair of damaged nerves. The results indicate that the gel was able to promote recovery of damaged nerves.
\end{abstract}

\section{Introduction}

The peripheral nervous system refers to all nerves other than the brain and spinal cord, including the ganglia, neural stem, plexus, and nerve terminals. Depending on the part of the central nervous system to which a nerve connects, the peripheral nervous system is categorized as either cranial or spinal, referring to its connection to the brain or spinal cord, respectively $[1,2]$. Peripheral nerve injuries are commonly observed in the clinic, often caused by fractures, mechanical trauma, or joint dislocation [3]. Injury to peripheral nerves is accompanied by a series of neurological lesions, which can cause the body to lose some or all of its motor, sensory, or autonomic function. Due to interruption of axon continuity, peripheral nerve damage also leads to neurofibrosis at the distal end of the lesion [4]. Therefore, adequate functional recovery after peripheral nerve injury remains a clinical challenge. Over the past ten years, use of microsurgery to treat damaged peripheral nerves has often failed to achieve the expected results due to the complex microenvironment in which the damaged nerves exist.
In addition to microsurgery, the local administration of suitable medications to nerve-damaged tissue may also be an effective and economical treatment [5].

Resveratrol (RSV) is a polyphenol of the plant antitoxin family, which can be found in a number of plants, such as peanuts, grapes, and blueberries. Studies over recent years have found that RSV has a wide range of biological effects, including being anti-inflammatory and with neuroprotective properties and protecting against oxidative stress and cancer [6-9]. The ability of RSV to reduce oxidative stress and mitochondrial dysfunction in Parkinson's disease models has been previously demonstrated by researchers [10]. In animal models, RSV can also reduce sciatic nerve injury caused by chronic crush injuries by regulation of an IGF-1-mediated immune response [11].

Despite the potential health benefits of RSV, its low solubility, slow rate of dissolution, and poor bioavailability have resulted in its limited clinical use. A number of strategies have been developed to counteract this problem. Liposomes are a class of artificial vesicles with a particle size in the nanometer range that can be produced from natural phospholipids and 
cholesterol. Due to the size, amphiphilic nature, and biocompatibility of liposomes, they represent a promising drug delivery system that can be used to increase drug solubility and bioavailability [12-14]. Liposomes have a bilayered structure; the core of which can contain hydrophilic compounds, with hydrophobic compounds contained within the bilayer membrane. Compounds encapsulated by liposomes tend to gradually diffuse from the bilayer into external liquids.

With the help of a local drug delivery system, the drug is concentrated on the target site, so the effectiveness and safety of its treatment are improved. The drug-encapsulated polymer-based hydrogel can be used locally to generate sufficient drug concentration [15]. And the thermal gel based of PLGA-PEG-PLGA has good biocompatibility and biodegradability, so its application potential in medical applications has attracted much attention. This hydrogel is in the form of a sol at room temperature and turns into a gel at body temperature, which makes it injectable and has been used as a sustained-release matrix for many drugs $[16,17]$. Since this type of hydrogel contains a lot of water, the small molecule drug contained in it will be released quickly in the initial stage, which is a burst release [18-20]. In the combined system of liposomes and hydrogels, liposomes can lower the burst release of hydrogels, so that the mixed system can achieve a sustained release effect, thereby increasing the bioavailability of the drug while reducing any adverse side effects $[21,22]$.

In the present study, RSV-loaded liposomes (RSV-Lips) were combined with a temperature-sensitive hydrogel to prepare a mixed drug delivery system for the continuous and stable release of drugs with improved drug utilization. Here, the mixed hydrogel was administered to a rat sciatic nerve injury to evaluate its repair capability of damaged nerves.

\section{Materials and Methods}

2.1. Materials and Animals. Resveratrol (98.5\% pure) was purchased from Alighting Reagent Company (Shanghai, China). Phospholipid was provided by Yuncheng Biochemical Company (Wuhan, China). Cholesterol and FITC (C6) were obtained from Sigma-Aldrich (USA). PLGA-PEGPLGA was synthesized in our own laboratory.

Chloroform and methanol were acquired from Sinopharm Chemical Reagent. Chromatographic grade acetonitrile was provided by Merck reagents (USA). Ultrapure water was prepared in the laboratory. All other reagents used were of analytical grade.

Sprague Dawley rats (200-222 g) were obtained from the Hubei University of Chinese Medicine Animal Center (Wuhan, China). All animal experiments were conducted strictly in accordance with the requirements of the "Principle of Animal Protection for Animal Experiments", and the experimental protocols were approved by the Animal Ethics Committee of the Medical University of Hubei University of Chinese Medicine.

2.2. Preparation and Optimization of RSV-Lips. Liposomes were prepared using a thin film dispersion technique. Briefly, resveratrol, phosphatidylcholine, and cholesterol in a $1: 25: 5$ ratio were dissolved in a mixed solution of chloroform and methanol (1:1 ratio) which was added to a round bottom flask and then dried using a rotary evaporator (RE52cs, Yali-wing Biochemical, Shanghai China) to form a thin lipid film. The dried film was then hydrated using $10 \mathrm{ml}$ PBS $(300 \mathrm{mM})$ at $35^{\circ} \mathrm{C}$ for $0.5 \mathrm{~h}$. The lipid dispersion was sonicated at $250 \mathrm{~W}$ for 10 min using an ultrasound probe (JY92 ultrasonic cell crusher, Xinzhi Institute of Scientific Instruments, Ningbo, China). Free resveratrol was removed by filtration through a $0.45 \mu \mathrm{m}$ polycarbonate microporous membrane. The prepared liposomes containing drugs were stored in a refrigerator at $4^{\circ} \mathrm{C}$.

A response surface method is a commonly used statistical methodology for optimizing experimental conditions. It identifies the relationship between variables and response to obtain the most appropriate conditions for preparation [23]. In the present study, a Box-Behnken Design (BBD), a common response surface methodology, was used to optimize the formulation of RSV-Lips $[24,25]$. The weight ratio of phospholipid to cholesterol, the weight ratio of phospholipid to drug, and the ultrasonic power value were used as independent variables, while the entrapment efficiency and drug loading capacity were used as response values.

2.3. Measurement of Entrapment Efficiency and Drug Loading Capacity. The measurement of encapsulation efficiency (EE) was accomplished through the use of microcolumn centrifugation. A $0.2 \mathrm{ml}$ aliquot of drug-containing liposome mixed solution was pipetted onto the top of a microseparation column (diameter $10 \mathrm{~mm} \times$ length $50 \mathrm{~mm}$, containing $1 \mathrm{~g}$ SephadexG-50 beads), to which $0.2 \mathrm{ml}$ PBS was added as the eluent and then centrifuge the column at $1000 \mathrm{~g}$ for 5 minutes. Repeated the centrifugation three times, adding new $0.2 \mathrm{ml}$ PBS eluent each time. Collected the eluate from three centrifugations into a $10 \mathrm{ml}$ volumetric flask, an appropriate volume of methanol was added, and the mixture was demulsified with ultrasound, prior to the addition of methanol to obtain a test solution. The solution was filtered through a $0.45 \mu \mathrm{m}$ microporous membrane and analyzed using high performance liquid chromatography. The following chromatographic detection conditions were selected. Methanol/water $(65: 35)$ mixed solvent as mobile phase in a C18 column was used, with a detection wavelength of $305 \mathrm{~nm}$. The following equation was used to calculate EE\% and drug loading capacity (DL)\%:

$$
\mathrm{EE} \%=\frac{\mathrm{Me}}{\mathrm{Mt}} \times 100 \%,
$$

where Me represents the quantity of encapsulated drug, and Mt the total quantity of drug.

$$
\mathrm{DL} \%=\frac{\mathrm{Me}}{W} \times 100 \%,
$$

where Me represents the quantity of encapsulated drug, and $W$ represents the quality of raw materials and excipients within the preparation. 


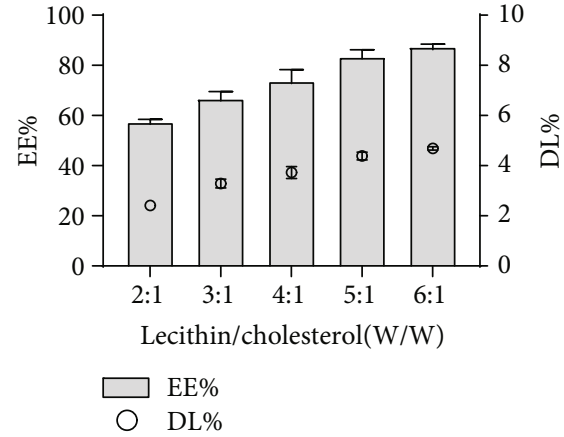

(a)

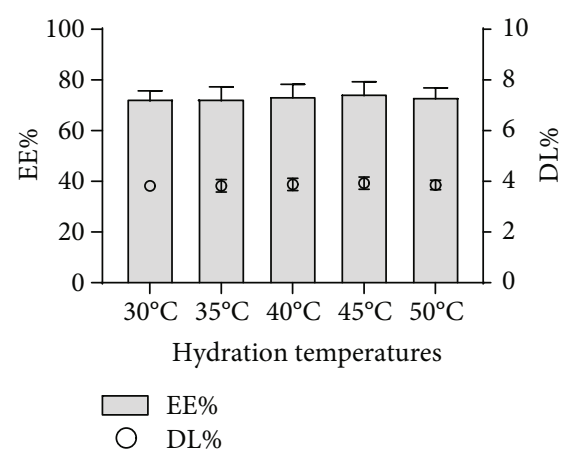

(c)

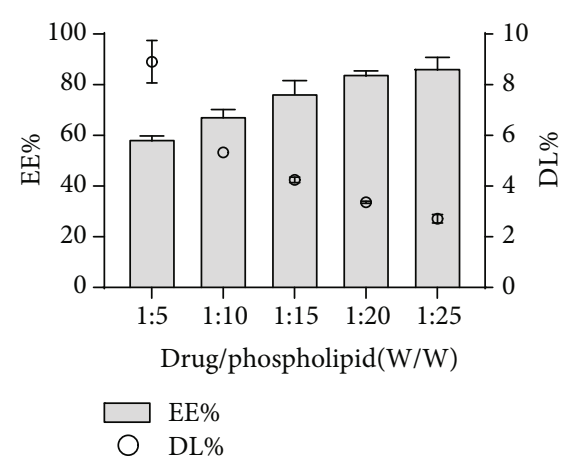

(b)

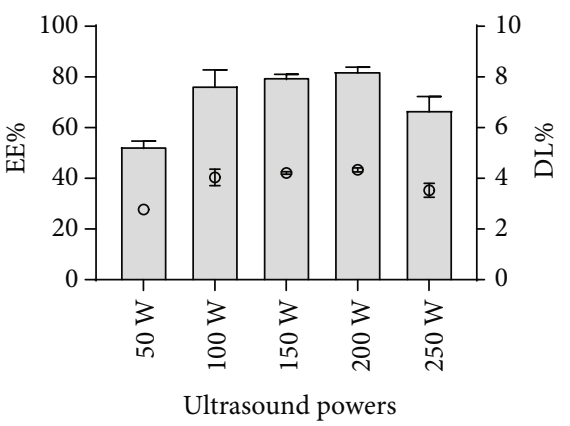

$\square \mathrm{EE} \%$

O DL\%

(d)

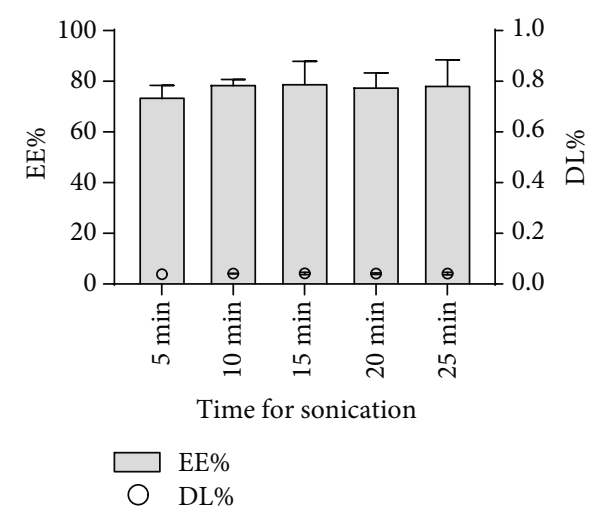

(e)

FIgURE 1: The results of the single factor experiment.

TABLE 1: BBD response surface experiment design factor level table.

\begin{tabular}{lccc}
\hline & \multicolumn{3}{c}{ Levels } \\
& -1 & 0 & +1 \\
\hline $\mathrm{X} 1$ & $4: 1$ & $5: 1$ & $6: 1$ \\
$\mathrm{X} 2$ & $1: 15$ & $1: 20$ & $1: 25$ \\
$\mathrm{X} 3$ & 100 & 150 & 200 \\
\hline
\end{tabular}

2.4. Preparation of Liposome Temperature-Sensitive Gel. Resveratrol liposome temperature-sensitive gel (RSV-Lips-Gel) was prepared using a simple mixing technique. Briefly, the weighed PLGA-PEG-PLGA copolymer (20 wt $\%$ ) was added to the RSV-Lips suspension and incubated without stirring at $4^{\circ} \mathrm{C}$ until the polymer was completely dissolved. Subsequently, the hydrogel solution was placed into a $1 \mathrm{ml}$ syringe, and pressure was applied to force the hydrogel through a 25 gauge needle so as to evaluate injectability of the gel solution at $25^{\circ} \mathrm{C}$. Finally, the prepared temperature-sensitive hydrogel containing drug was stored in a refrigerator at $4^{\circ} \mathrm{C}$. Blank liposomes gel (Blank Lips-Gel) was prepared using the method described above, but without the inclusion of drug.

2.5. Evaluation of Particle Size and Zeta Potential. Particle size and zeta potential of the RSV-Lips and RSV-Lips-Gel were measured using a dynamic laser scattering instrument (Malvern Zetasizer Nano ZS90, UK). Prior to measurement, the hydrogel sample was diluted with PBS solution to obtain a concentration of $10 v / v \%$ and filtered through an aqueous 
TABle 2: Box-Behnken response surface experiment design and result table.

\begin{tabular}{lcccccc}
\hline & \multicolumn{3}{c}{$\begin{array}{c}\text { Independent } \\
\text { variables }\end{array}$} & \multicolumn{3}{c}{ Dependent variables } \\
& $\mathrm{X} 1$ & $\mathrm{X} 2$ & $\mathrm{X} 3$ & $\mathrm{Y} 1$ & $\mathrm{Y} 2$ & $Y$ \\
\hline 1 & -1 & -1 & 0 & 65.7 & 3.33 & 34.52 \\
2 & 1 & -1 & 0 & 63.7 & 3.23 & 33.47 \\
3 & -1 & 1 & 0 & 78.2 & 2.42 & 40.29 \\
4 & 1 & 1 & 0 & 81.5 & 2.7 & 42.08 \\
5 & -1 & 0 & -1 & 79.4 & 3.05 & 41.21 \\
6 & 1 & 0 & -1 & 78.7 & 3.24 & 40.98 \\
7 & -1 & 0 & 1 & 77.2 & 2.97 & 40.10 \\
8 & 1 & 0 & 1 & 81.5 & 3.35 & 42.42 \\
9 & 0 & -1 & -1 & 65.4 & 3.44 & 34.43 \\
10 & 0 & 1 & -1 & 71.6 & 2.31 & 36.95 \\
11 & 0 & -1 & 1 & 70.6 & 3.71 & 37.14 \\
12 & 0 & 1 & 1 & 78.9 & 2.54 & 40.71 \\
13 & 0 & 0 & 0 & 87.9 & 3.52 & 45.71 \\
14 & 0 & 0 & 0 & 89.7 & 3.59 & 46.66 \\
15 & 0 & 0 & 0 & 90.5 & 3.62 & 47.07 \\
16 & 0 & 0 & 0 & 88.6 & 3.54 & 46.05 \\
17 & 0 & 0 & 0 & 93.2 & 3.73 & 48.44 \\
\hline
\end{tabular}

microporous membrane with a pore size of $0.45 \mu \mathrm{m}$. Set the detection temperature of the instrument to $25^{\circ} \mathrm{C}$ and the equilibration time to 120 seconds.

2.6. Rheological Testing. The rheological properties of the Blank Lips-Gel and RSV-Lips-Gel were measured using a rotational rheometer (HAAKE 6000, Thermo Scientific, Germany). The temperature was gradually raised from $25^{\circ} \mathrm{C}$ to $40^{\circ} \mathrm{C}$ at $1^{\circ} \mathrm{C} / \mathrm{min}$ to determine its viscosity at different temperatures.

2.7. Thermosensitivity Evaluation of RSV-Lips-Gel. The phase transition temperature and inversion time of the blank gel and gel containing RSV were measured using a tube inversion method. Briefly, a $2 \mathrm{ml}$ sample of each group was added to a $10 \mathrm{~cm}$ long $1.5 \mathrm{~cm}$ diameter test tube. These test tubes were then placed in a water bath, and the temperature raised gradually, from $25^{\circ} \mathrm{C}$ to $40^{\circ} \mathrm{C}$. At each temperature, the gel samples, after equilibration for 3 minutes, were tested to observe the flowability of each sample following inversion of the test tube. The temperature at which the sample became immobile was recorded as the gelation temperature.

2.8. Biocompatibility of Gel In Vivo. A $0.5 \mathrm{ml}$ aliquot of blank gel and an aliquot of gel containing drugs were subcutaneously injected into the rear of Kunming mice (female, $20-22 \mathrm{~g}$ ). The mice were sacrificed at predetermined time points ( 3 and 7 days) and then the skin from the site of injection harvested and fixed in $4.0 \%(w / v)$ paraformaldehyde. Sections of the skin were stained with hematoxylin and eosin (HE) for pathological analysis.
2.9. In Vitro Drug Release Study. A dialysis membrane (10$15 \mathrm{kDa}$ molecular weight cut-off, Sinopharm Reagent, China) was used to investigate the release of drug from RSV-LipsGel and RSV-loaded hydrogel (RSV-Gel). Precisely, $2 \mathrm{ml}$ of the RSV-Lips-Gel were placed into a dialysis bag then dialyzed against $100 \mathrm{ml}$ PBS $(0.01 \mathrm{M})$, to which $0.5 \%$ Tween 80 was added. Throughout the release study, the rate of stirring was adjusted to $75 \mathrm{rpm}$ and temperature to $37 \pm 0.5^{\circ} \mathrm{C}$. At intervals of 1 hour, $1 \mathrm{ml}$ of dialysate was removed and replaced with fresh solution of the same volume at a temperature of $37^{\circ} \mathrm{C}$. HPLC, as described in Section 2.2, was used to measure drug concentration in the release medium.

2.10. Evaluation of In Vivo Efficacy. In vivo pharmacological activity was assessed using a rat sciatic nerve crush injury model $[3,26]$. Briefly, rats were anesthetized by intraperitoneal injection of $20 \%$ urose solution $(200 \mathrm{mg} / \mathrm{kg})$, and the right sciatic nerve was isolated by blunt dissection. Hemostatic forceps were used to create a crush injury to the nerve, the force of which was measured using a membrane pressure sensor (RFP-602, Yubo Intelligent Technology, China). The pressure sensor was calibrated using a stress detector (DT-800, DataTaker, Australia) prior to the experiment. The hemostatic forceps were placed vertically to compress the middle section of the sciatic nerve with a force of $5 \mathrm{~N}$ for $8 \mathrm{~s}$, with compression repeated 3 times at $5 \mathrm{~s}$ intervals. Following this procedure, the rats were randomly allocated into four groups $(n=5$ per group). One group was administered normal saline as a model control group, another provided free RSV $(10 \mathrm{mg} / \mathrm{kg})$, and the remaining two groups treated with RSV-Lips-Gel $(5 \mathrm{mg} / \mathrm{kg}$ and $10 \mathrm{mg} / \mathrm{kg})$. In addition, a sham group, in which the sciatic nerve was exposed, but not crushed, was also included. The drug was injected into the injured nerve site immediately after the procedure. Completion of the procedure was termed day 0. Subsequent intramuscular injections were performed every 5 days; the experiment terminated after 25 days. Animal welfare was fully considered during the entire experiment. The experimental animals had ad libitum access to food and drinking water.

2.11. Sciatic Functional Index and Thermal Paw Withdrawal Latency. Sciatic nerve functional index (SFI) was calculated by analysis of walking trajectory. The following method established by Fey et al. [27] was used with appropriate adjustments. A bespoke device in the form of a channel $(6 \times 8 \times 40 \mathrm{~cm})$ was used to record the footprints of the rats. White paper was placed underneath, and the hind legs of the rats painted with pigment prior to recording. As the rat passed the channel, the posterior foot prints were printed on the paper. The rats were allowed to pass freely through the channel before recording to allow the rat to adapt to the test environment.

Tests continued until five measurable footprints were recorded, from which, several parameters were measured: (i) print length (PL: distance from the top of the third toe to the heel); (ii) toe spread (TW: distance between the first and the fifth toe), and (iii) intermediary toe spread (IT: 


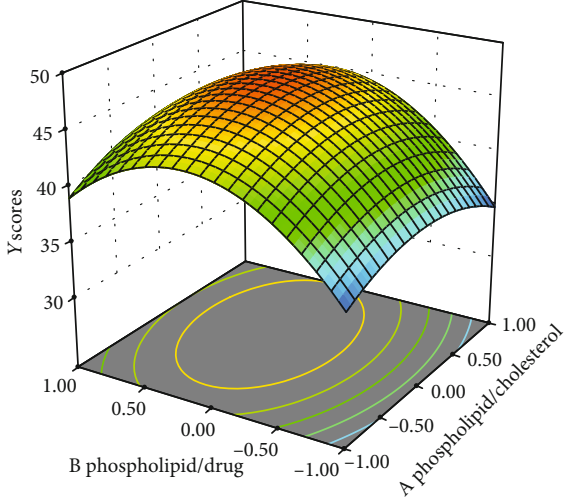

(a)

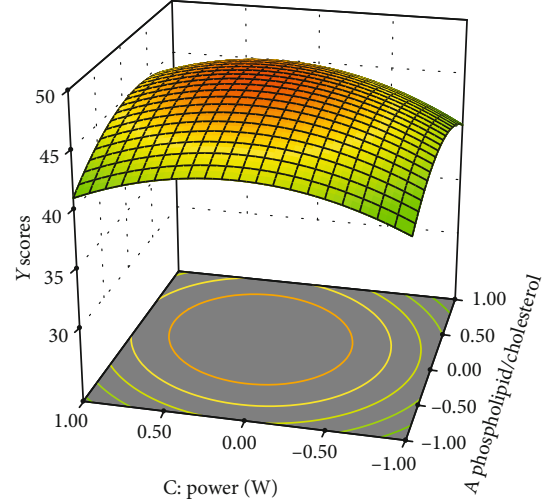

(b)

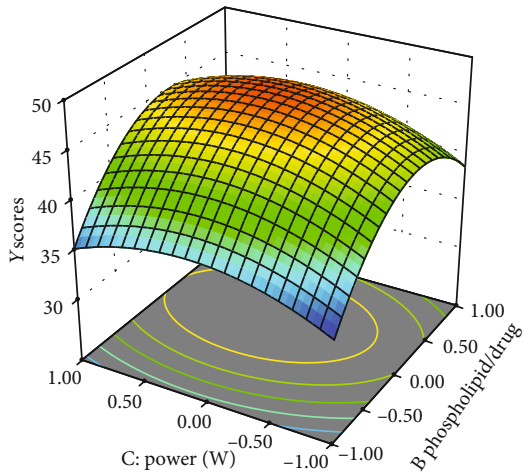

(c)

Figure 2: Three-dimensional response surface curve. (a) Response surface graphs of phospholipid/drug and phospholipid/cholesterol. (b) Response surface graphs of phospholipid/cholesterol and power. (c) Response surface graphs of phospholipid/drug and power.

TABLE 3: Analysis of variance of quadratic multiple regression model of response variables.

\begin{tabular}{lccccc}
\hline Source & Sum of squares & $\mathrm{df}$ & Mean square & $F$ value & $p$ value \\
\hline Model & 331.55 & 9 & 36.84 & 14.28 & 0.001 \\
$\mathrm{~A}$ & 1.00 & 1 & 1.00 & 0.39 & 0.5531 \\
$\mathrm{~B}$ & 52.38 & 1 & 52.38 & 20.3 & 0.0028 \\
$\mathrm{C}$ & 5.78 & 1 & 5.78 & 2.24 & 0.1781 \\
$\mathrm{AB}$ & 2.02 & 1 & 2.02 & 0.78 & 0.406 \\
$\mathrm{AC}$ & 1.63 & 1 & 1.63 & 0.63 & 0.4534 \\
$\mathrm{BC}$ & 0.28 & 1 & 0.28 & 0.11 & 0.7534 \\
$\mathrm{~A}^{2}$ & 29.86 & 1 & 29.86 & 11.57 & 0.0114 \\
$\mathrm{~B}^{2}$ & 179.71 & 1 & 179.71 & 69.64 & $<0.0001$ \\
$\mathrm{C}^{2}$ & 36.53 & 1 & 36.53 & 14.16 & 0.0071 \\
Residual & 18.06 & 7 & 2.58 & & \\
Lack of fit & 13.53 & 3 & 4.51 & 3.98 & 0.1076 \\
Pure error & 4.53 & 4 & 1.13 & & \\
Cor total & 349.62 & 16 & & & \\
\hline
\end{tabular}

distance from the second to the fourth toe). The three measures were recorded from the normal foot (NPL, NTW, and NIT) and from the foot affected by the crush injury (EPL, ETW, and EIT). SFI was calculated using the formula SFI =
TABLE 4: Results of three batches of verification experiments.

\begin{tabular}{lcccc}
\hline No. & EE\% & DL\% & $Y$ value & Error\% \\
\hline 1 & 86.6 & 3.3 & 44.9 & \\
2 & 83.4 & 3.2 & 43.3 & $4.6 \%$ \\
3 & 86.5 & 3.3 & 44.9 & \\
\hline
\end{tabular}

$-38.3 \times(\mathrm{EPL}-\mathrm{NPL}) / \mathrm{NPL}+109.5 \times(\mathrm{ETW}-\mathrm{NTW}) / \mathrm{NTW}$ $+13.3 \times(\mathrm{EIT}-\mathrm{NIT}) / \mathrm{NIT}-8.8$.

In normal rats, the SFI score is close to 0 . Lower scores in the surgical model represented more severe injury, a score of 100 indicating complete injury.

An animal hot plate was used to detect latency of paw withdrawal from heat (PWTL). The animals were placed on hot plates adjusted to $50 \pm 0.5^{\circ} \mathrm{C}$ (YLS-6B Hot-Plate, Zheng hua Biological Instruments, China). Hyperalgesia was assessed by measuring the latency of paw licking or jumping reactions as an indicator of pain. The mean of the three measurements was recorded as the value of the withdrawal response, with an interval of 10 minutes between each measurement. In order to reduce burns, a cut-off time not greater than 50 seconds was used.

Animal behavioral experiments were performed twice a day prior to surgery and every 5 days afterwards, with a total of 6 tests performed. 


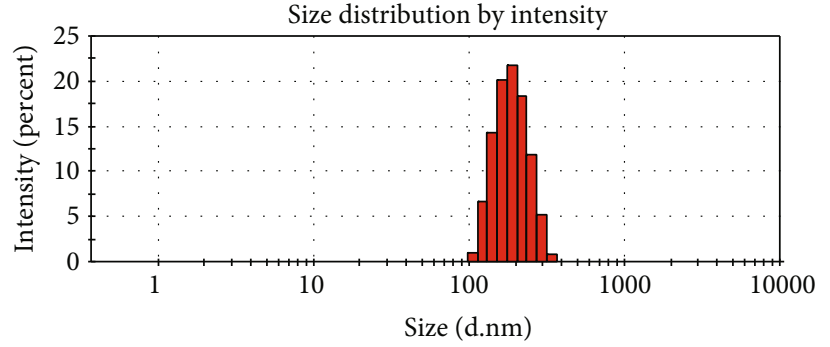

(a)

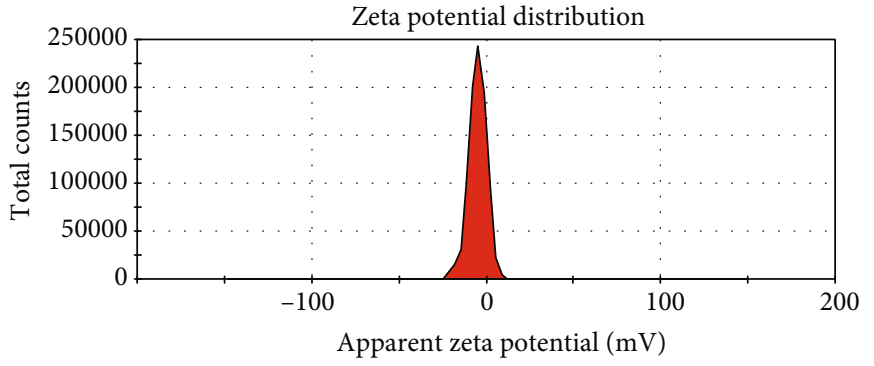

(c)

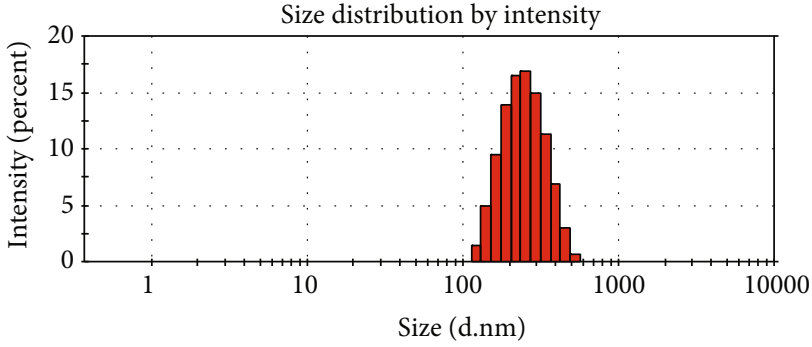

(b)

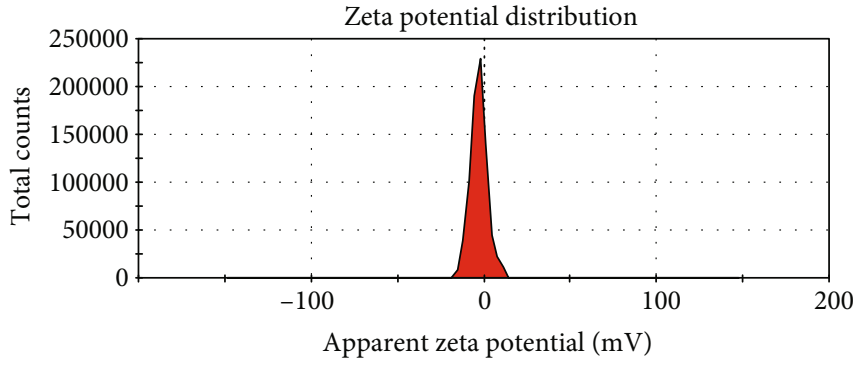

(d)

Figure 3: Particle size distribution of RSV-Lips and RSV-Lips-Gel. (a, c) RSV-Lips; (b, d) RSV-Lips-Gel.

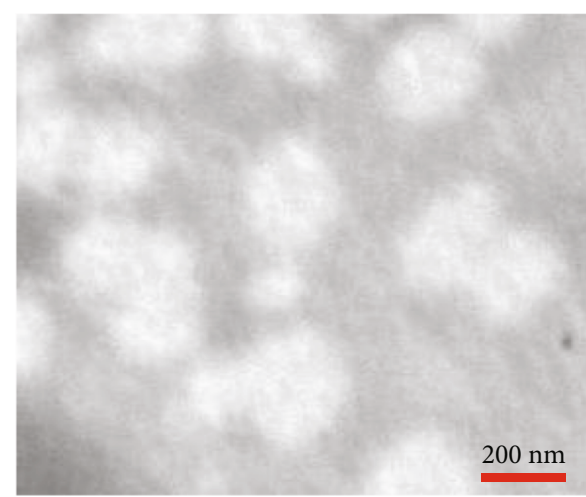

(a)

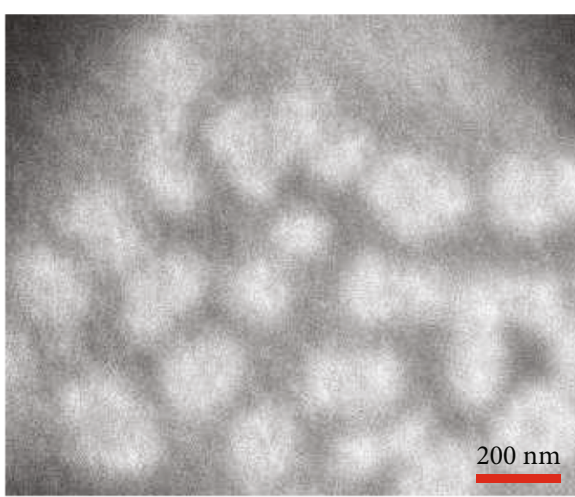

(b)

Figure 4: TEM observation images of RSV-Lips and RSV-Lips-Gel (×20 k). (a) RSV-Lips; (b) RSV-Lips-gel.

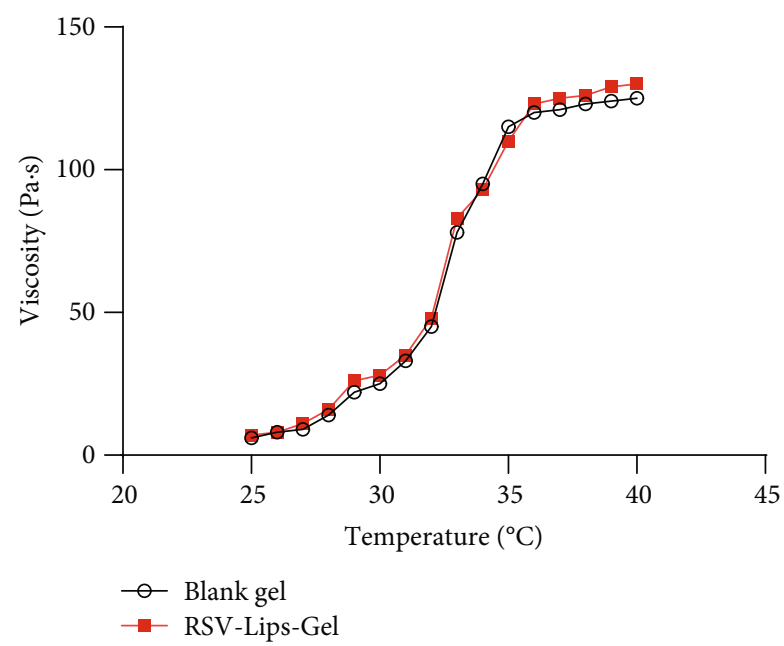

FIGURE 5: Viscosity of blank gel and RSV-Lips-Gel.
2.12. Histopathological Examination. All rats were sacrificed after behavioral testing on day 28. The sciatic nerve was isolated for observation of pathological sections. The gastrocnemius muscle was isolated, weighed, and then sectioned for observation by microscopy. The sciatic nerve was dissected at a distance of $0.5 \mathrm{~cm}$ from the distal site of injury, then fixed in paraformaldehyde at $4^{\circ} \mathrm{C}$ overnight. It was embedded in resin then sliced into half-thin $(500 \mathrm{~nm})$ sections, stained with $1 \%$ toluidine blue, and observed using a light microscope (TS-100, Nikon, Japan).

2.13. Statistical Analysis. All data represent means \pm SD. ANOVA was used to compare the significance of differences between groups. $p$ values $<0.05$ were considered statistically significant.

\section{Results and Discussion}

3.1. Preparation and Characterization of RSV-Lips. In order to compare thin film dispersion methods, ethanol injection 


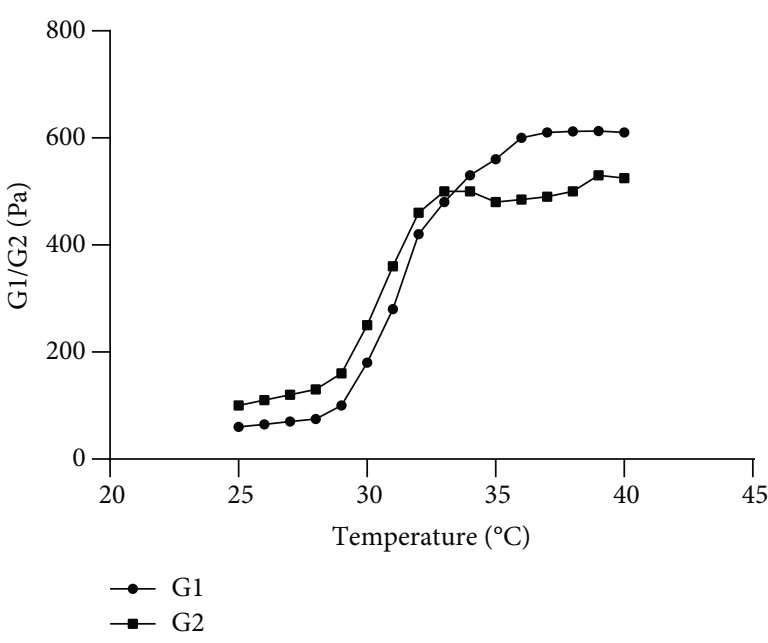

(a)

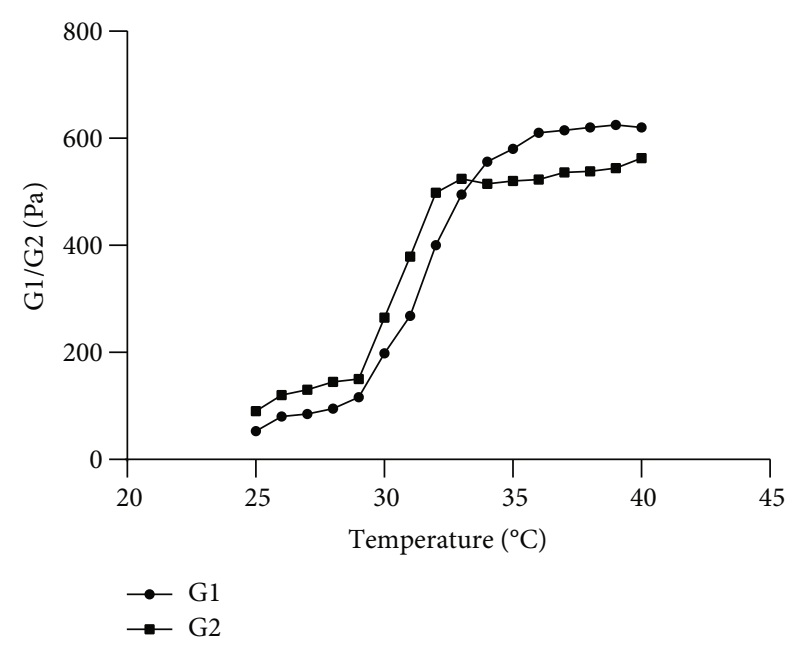

(b)

Figure 6: Characterization of phase transition process. (a) Blank gel; (b) RSV-Lips-Gel.

method and inverse evaporation method were evaluated. The latter two methods resulted in low EE or unstable liposomes with a large particle size distribution. It can be seen that the thin film dispersion method was more suitable for lipophilic substances such as RSV. Five different single factors that may have an impact on the quality of liposomal preparation were selected, including the ratio of phospholipid to cholesterol $(2: 1,3: 1,4: 1,5: 1$, and $6: 1)$, ratio of drug to phospholipid $(1: 5,1: 10,1: 15,1: 20$, and $1: 25)$, hydration temperature $\left(30,35,40,45\right.$, and $\left.50^{\circ} \mathrm{C}\right)$, ultrasonic power $(50$, $100,150,200$, and $250 \mathrm{~W})$, and duration of sonication $(5,10$, 15,20 , and 25 minutes) for single factor experiments, with $\mathrm{EE}$ and DL of liposomes as dependent variables. The results of the single factor experiments are displayed in Figure 1. Among the five factors examined, the three factors, phospholipid to cholesterol ratio, drug-lipid ratio, and ultrasonic power, all provided a significant effect on encapsulation rate and drug loading. Specifically, encapsulation rate increased with increasing phospholipid ratio in the range $2: 1-6: 1$. At a drug-lipid ratio between $1: 5$ and $1: 25$, increasing druglipid ratio caused a gradual increase in encapsulation ratio. Although the drug loading rate in the range of $1: 5$ and $1: 10$ is higher than other ranges, the encapsulation efficiency in this range is less than $70 \%$. The range of investigation of ultrasonic power was between $50 \mathrm{~W}$ and $250 \mathrm{~W}$, which had a significant effect on the rate of encapsulation of liposomes, the trend of which first increased then decreased. Maximum rate of encapsulation was achieved at an ultrasonic power of between $150 \mathrm{~W}$ and $200 \mathrm{~W}$. The other factors considered, hydration temperature and sonication time, had no significant effect on rate of encapsulation and drug loading. Considering preparation time and energy consumption, a hydration temperature of $40^{\circ} \mathrm{C}$ and sonication time of $15 \mathrm{~min}$ were selected for subsequent optimization experiments.

Single-variable test results were used to determine the range of independent variables. Finally, the weight ratio of phospholipid to cholesterol (X1), the weight ratio of phospholipid to drug (X2), and the ultrasonic power value (X3) were key factors. In order to comprehensively consider the influence of factors on EE and DL, we define the $Y$ value here, and the calculation formula is $Y=0.5 \times \mathrm{EE}+0.5 \times \mathrm{DL}$. The range and center point values of the three independent variables involved in this study are listed in Table 1. The center point was tested five times to identify systematic errors. Experimental data are displayed in Table 2. The experimental design experiments were performed in random order. Analysis of experimental data was performed using DesignExpert software (version 8.05). Data were analyzed by ANOVA. The 3D response surface produced by DesignExpert v8.0.6 was used to explain the interaction between variables, the result of which is presented in Figure 2. The quadratic multiple regression model and analysis of variance based on the response variables are shown in Table 3. The degree of influence on the response value can be determined by observing the inclination of the $3 \mathrm{D}$ response surface. The interaction was more significant for larger values of slope. The Design-Expert v8.0.6 software was used to analyze the experimental results, with a ternary quadratic regression equation of score $Y$ and ratio of phosphorus/gallbladder (X1), drug-lipid ratio (X2), and ultrasonic power (X3). The equation is $Y=46.79+0.35 \times \mathrm{X} 1+2.56 \times \mathrm{X} 2+0.85 \times \mathrm{X} 3+$ $0.71 \times \mathrm{X} 1 \mathrm{X} 2+0.64 \times \mathrm{X} 1 \mathrm{X} 3+0.26 \times \mathrm{X} 2 \mathrm{X} 3-2.66 \times \mathrm{X}^{2}-$ $6.53 \times \mathrm{X} 2^{2}-2.95 \times \mathrm{X}^{2}$.

Analysis of variance indicated that the $p$ value of the model was $<0.01$, indicating that the regression model established was extremely significant, with a $p$ value of the mismatch term $>0.05$ and so not significant, indicating that the model could be used for data analysis in the present study. According to the $p$ values in the variance table, the order of influence of single factors in the model was $\mathrm{X} 2>\mathrm{X} 3>\mathrm{X} 1$, among which factor $\mathrm{X} 2$ was extremely significant, with the impact of $\mathrm{X} 1$ and $\mathrm{X} 3$ not significant. The interaction terms $\mathrm{X} 1 \mathrm{X} 2, \mathrm{X} 1 \mathrm{X} 3$, and $\mathrm{X} 2 \mathrm{X} 3$ affected the comprehensive score $Y$ , although none were significant. The effects of $\mathrm{X}^{2}, \mathrm{X} 2^{2}$, and $\mathrm{X} 3^{2}$ in the second term were all significant. It can be seen from the response surface graph that the drug-lipid ratio and 


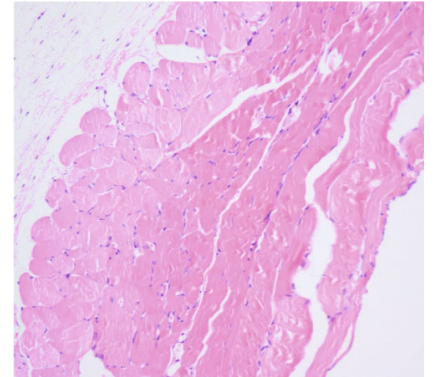

(a)

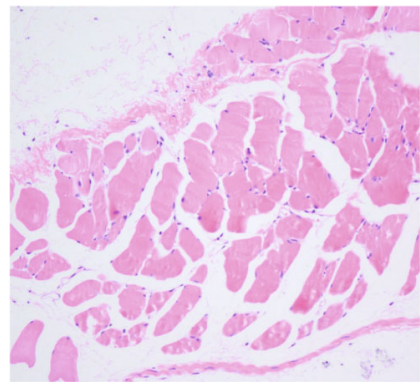

(c)

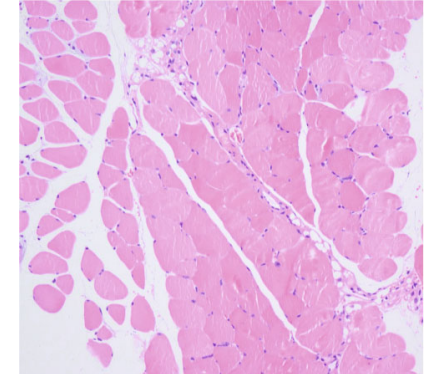

(b)

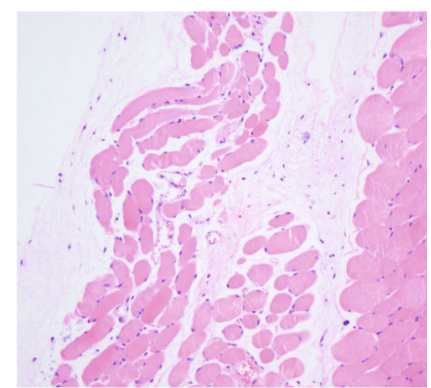

(d)

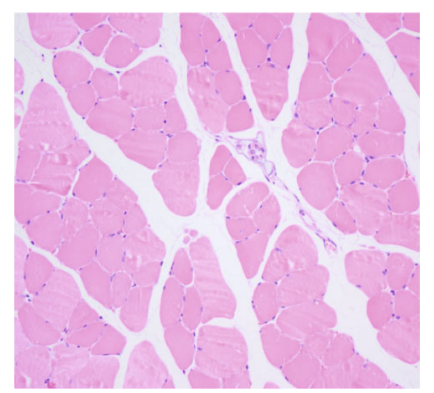

(e)

Figure 7: Results of pathological section of Biocompatibility test. (a) Normal, (b) blank gel (1 d), (c) blank gel (5 d), (d) RSV-Lips-Gel (1 d), (e) RSVLips-Gel (5 d).

ultrasonic power had a substantial impact on score $Y$; the impact surface graph is relatively steep, consistent with the results of analysis of variance.

Larger values of $Y$ represent better quality of liposomes. The regression equation is at its maximum value when the optimal conditions are as follows: ratio of phosphorus to gallbladder: $5.1: 1$; ratio of drug to lipid: $1: 21$; and ultrasonic power: $158.5 \mathrm{~W}$. Subsequently, three batches of samples were prepared using the optimal conditions, and encapsulation efficiency and drug loading were determined. The test results are shown in Table 4 . The actual measurement results were within a 5\% error from those predicted by the model, indicating that the model provided a good predictive fit and that the data obtained was valid and reliable.

3.2. Preparation and Characterization of RSV-Lips-Gel. In order to adjust the viscosity and osmotic pressure of RSVLips-Gel, mannitol and sodium chloride were added to the formulation. Through orthogonal testing, an optimized formulation of RSV-Lips-Gel was finally determined, namely,
RSV-Lips concentration: 50\%; copolymer concentration: $20 \%$; mannitol concentration: $1 \%$; sodium chloride concentration: $0.9 \%$.

The encapsulation rates of the prepared RSV-Lips were all greater than $80 \%$. No significant change in encapsulation rate was observed after temperature-sensitive gels were used in the fabrication of the liposomes. It can be seen from Figure 3 that RSV-Lips initially had a particle diameter of $188.8 \pm 9.7 \mathrm{~nm}$ and a zeta potential of -2.95 $\pm 0.23 \mathrm{mv}$. Using a temperature-sensitive gel, the diameter was $210.5 \pm 4.7 \mathrm{~nm}$ and zeta potential of $-4.73 \pm 0.16 \mathrm{mv}$, suggesting that the liposomes were larger with a decreased zeta potential. As displayed in Figure 4, the basic morphology of the liposomes did not significantly change after inclusion of a temperature-sensitive gel, the liposomes being essentially spherical as observed by projection imaging electron microscope. In summary, encapsulation efficiency, particle size, zeta potential, and appearance were not significantly affected by use of a temperaturesensitive gel. The results suggest that RSV was encapsulated in the gel in the form of liposomes. 


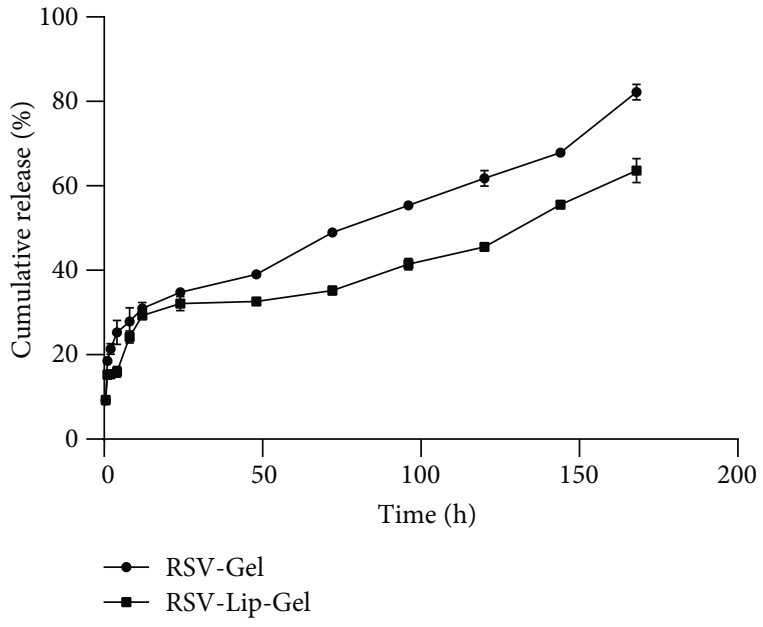

FIGURE 8: Results of in vitro release experiments.

The change in viscosity of Blank Lips-Gel and RSV-LipsGel with increasing temperature is presented in Figure 5. Both Blank Lips-Gel and RSV-Lips-Gel were less viscous below $30^{\circ} \mathrm{C}$, indicating that the gel was more fluid at low temperatures and suggesting that the addition of drugs did not greatly affect gel fluidity and thus was suitable for injection at room temperature. However, the viscosity of the gel increased sharply above $30^{\circ} \mathrm{C}$. At this temperature, the gel underwent a phase transition, from sol to gel. As shown in Figure 6, the elastic modulus (G1) and the viscosity modulus (G2) of the gel changed significantly as temperature increased. At temperatures higher than $30^{\circ} \mathrm{C}$, the detection value gradually increased, with G1 increasing greater than G2, indicating the start of the change from sol to gel state. The temperature at which G1 and G2 intersected is known as the gelling temperature. At temperatures higher than this value, changes in G1 and G2 tended to be gradual. In the two gelation tests, temperatures of $32.5^{\circ} \mathrm{C}$ and $33.2^{\circ} \mathrm{C}$ for gelation were recorded, indicating that incorporation of drug did not significantly change the gelation temperature of the thermosensitive gel. A more visual test of this phenomenon is the inverted test tube method to detect phase change. The liposome temperature-sensitive gel clearly formed a gel state when the temperature was approximately $37^{\circ} \mathrm{C}$, where the sample was unable to flow upon tube inversion, a temperature slightly higher than that indicated by the rheometer. At higher temperatures, interactions of the hydrophobic bonds between PLGA blocks of the triblock polymer are enhanced, leading to micelle aggregation and conversion to a gel state [28-30]. The results indicate that RSV encapsulation did not significantly affect the gel-forming properties of the hydrogel.

HE staining of tissue sections after subcutaneous injection of the thermogel are presented in Figure 7. One day after injection of the gel, a mild acute inflammatory response was visible in tissue sections compared with normal skin. Due to the gradual degradation of the hydrogel, tissue inflammation was significantly lower after 5 days than after 1 day. In addition, no skin tissue samples exhibited necrosis. The results

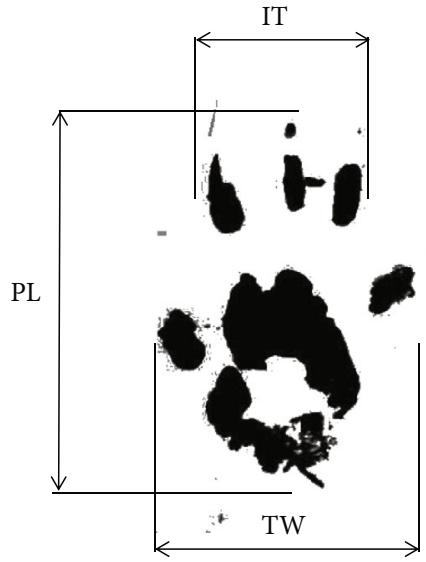

FIGURE 9: Schematic of SFI values (PL: distance from the top of the third toe to the heel; IT: distance from the second to the fourth toe; TS: distance between the first and the fifth toe).

demonstrate that the gel exhibited good biocompatibility in vivo.

3.3. In Vitro Drug Release Study. The drug release profiles of RSV-Gel and RSV-Lips-Gel formulations are displayed in Figure 8. Optimal conditions for drug release were determined from the equilibrium solubility of RSV. RSV has good solubility in PBS $(0.01 \mathrm{M})$ containing $0.5 \%$ Tween 80 . The release from RSV-Lips-Gel was significantly extended compared with RSV-Gel. The cumulative drug release from RSV-Lip-Gel was $32 \%$ after 24 hours, and $64 \%$ by the end of the experiment, considerably lower than the release from RSV-Gel. The reason is due to RSV-Lips-Gel forming a colloid under experimental conditions, with a firmer texture than RSV-Gel, which resists attack from aqueous solvents, resulting in a slow dissolution rate and a correspondingly longer drug release time. The in vitro release behavior of RSV-Lip-Gel follows that of the Ritger-Peppas model [31, 32]. The RSV-Lips and triblock polymer constitute a liposomal temperature-sensitive gel. RSV-Lips can be concentrated in the hydrophilic long chains of the polymer gel. As the gel matrix dissolves, the hydrophilic chain becomes fully exposed to the aqueous environment, and the encapsulated RSV-Lips is gradually released, with RSV in the liposomes diffusing into the aqueous environment. In vitro release from RSV-Lips-Gel is a combination of erosion and diffusion, that is, the release behavior is in accordance with the RitgerPeppas equation [31, 32]. From the 24-hour drug release curve, it can be seen that quantity released from RSV-LipsGel was lower than that of RSV-Gel, indicating that the burst phenomenon of the liposome temperature-sensitive gel was improved compared with the ordinary gel.

3.4. Evaluation of In Vivo Efficacy. Animal models of peripheral nerve injury are generally established in a rabbit or rat using the sciatic nerve, involving either complete or partial injury. Injury can be produced by extrusion, electric burns, freezing, acupuncture, or using a cross-section method [33, 34]. In the present study, rat sciatic nerve compression was used for the peripheral nerve injury model. This model is 


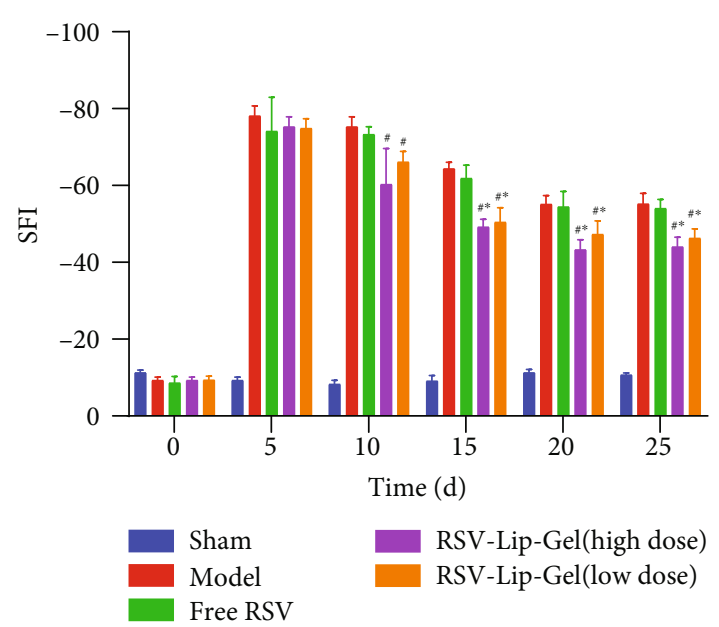

(a)

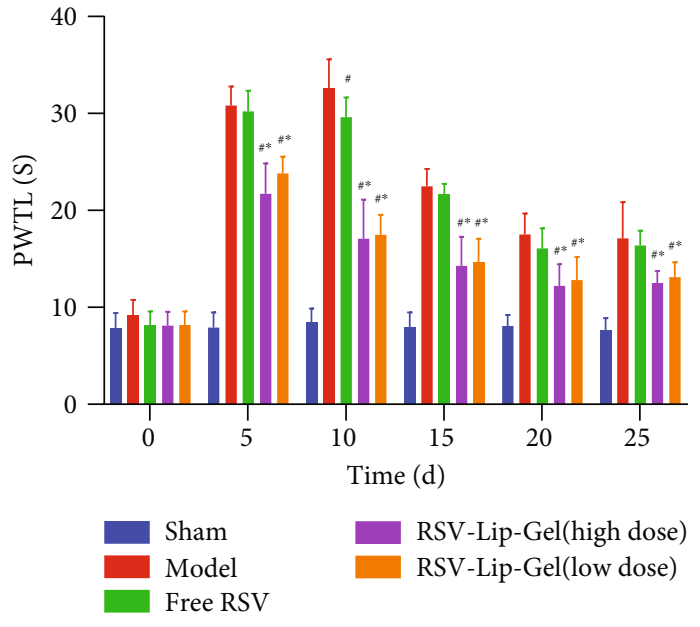

(b)

Figure 10: Test results of animal behavior experiments $\left({ }^{\#} p<0.05\right.$ compared with the model group; ${ }^{*} p<0.05$ compared with the free drug group).

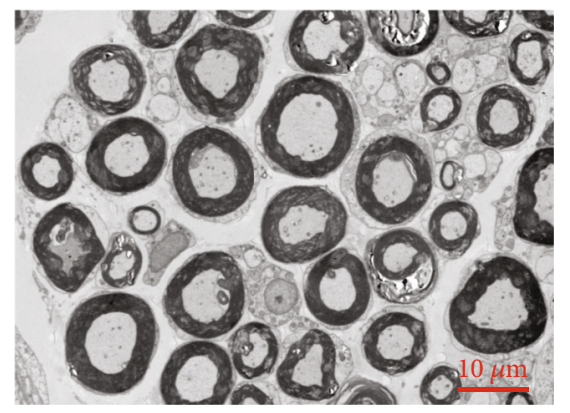

(a)

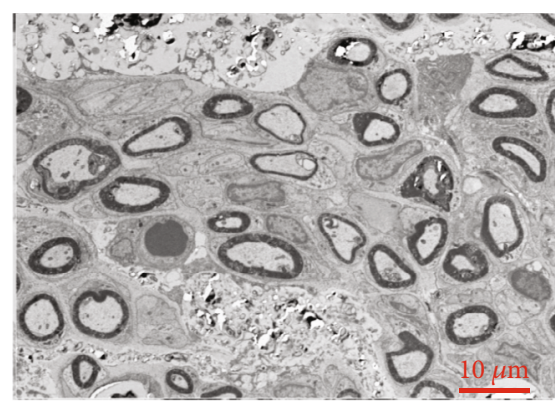

(c)

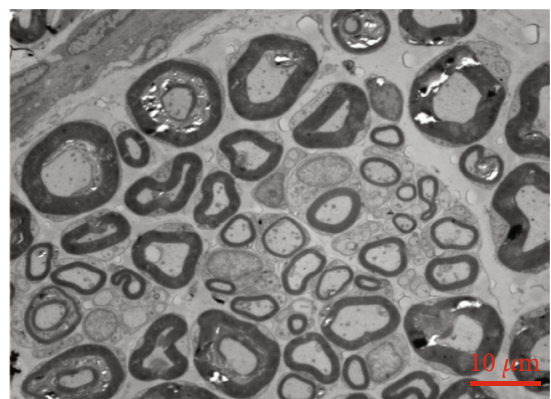

(e)

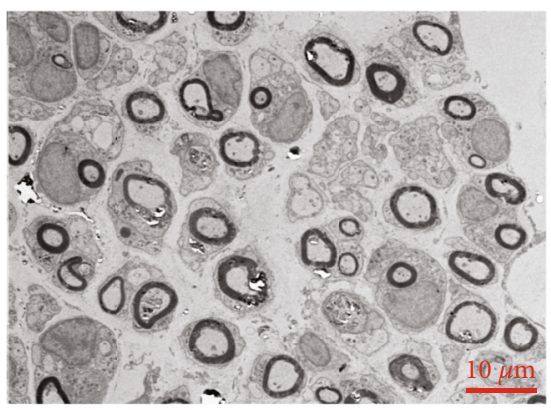

(b)

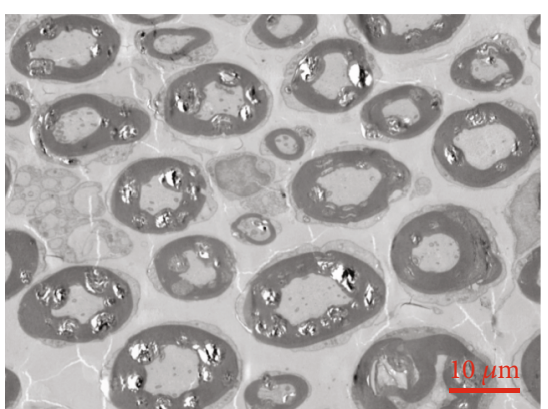

(d)

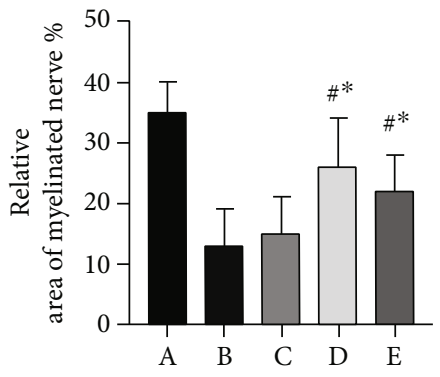

(f)

FiguRE 11: Observation of the sciatic nerve in a semithin section ((a) sham, (b) model, (c) free RSV, (d) RSV-Lip-Gel high dose, (e) RSV-LipGel low dose, ${ }^{*} p<0.05$ compared with the model; ${ }^{*} p<0.05$ compared with the free RSV). 
widely used in experimental studies of peripheral nerve regeneration and repair. It is simple to establish and interpret and has good reproducibility, with rapid postoperative recovery, and behavioral assessment of animals can be conducted within days of injury.

The toe measurement method in rats is displayed in Figure 9, and the results of animal behavior tests in Figure 10. SFI values of rats in each group prior to surgery (Figure 10(a)) were less than -10 , indicating that there were no differences, and nerve function of the animals in each group was normal. Compared with the sham group, the SFI value in each group after surgery was significantly different $(p<0.05)$. SFI values were measured at $10,15,20$, and 25 days after surgery and compared with the value measured at 5 days. SFI values in the high- and low-dose groups at each time point after 10 days were significantly different from that of the model group $(p<0.05)$, and in the gel-dose group better than in the free drug group, differences that were statistically significant $(p<0.05)$. As shown in Figure 10(b), PWTL values prior to surgery were in the range $6 \mathrm{~s}-10 \mathrm{~s}$ across all groups, indicating that no differences were observed. Compared with the sham group, PWTL in each group increased significantly after surgery. PWTL values in each group decreased with time. Compared with the model group, the PWTL values in each group were significantly different after 10 days $(p<0.05)$. The PWTL value at the 25 -day time point in the high-dose group was significantly different from the free drug group $(p<0.05)$.

Animal behavioral assessments included tests for SFI and PWTL. SFI is a reliable indicator of nerve regeneration and functional recovery after sciatic nerve injury. PWTL measures the degree of pain using a hot plate, allowing more standardized experimentation and reduced experimental variation $[33,34]$. The results demonstrate that the RSV-Lips-Gel high-dose group had significantly increased SFI and reduced PWTL values compared with the model and free RSV groups, indicating that RSV-Lips-Gel assisted in the recovery of sciatic nerve function.

Figure 11 displays a semithin section of the sciatic nerve. It can be seen that the myelinated fibers of the sciatic nerve in all experimental groups except the sham group underwent significant degenerative changes, with some axons that had disappeared. The model group had a loose structure, with enlarged intercellular spaces, appearing vacuolated. The RSV-Lips-Gel high-dose group had myelinated nerve fibers that appeared oval or almost round, with cells that were more closely packed. In the low-dose group, the myelin fibers were loosely arranged, irregular in shape, with a thin myelin sheath and the presence of vacuoles. The cross-sectional area of myelinated fibers in the semithin sections was recorded across the entire visual field. Compared with the sham group, the number of myelinated nerve fibers in the model group was significantly lower which had the smallest total crosssectional area. The proportion of myelinated nerve fibers in the RSV-Lips-Gel high-dose group was higher, including in the RSV-Lips-Gel low-dose group. The relative area of myelinated fibers in the free RSV group was not statistically different from that in the model group. This suggests that the gel-administered group improved damaged sciatic nerve morphology at a particular dose. The change in SFI values was highly correlated with histomorphology of the sciatic nerve crush injury model. The RSV-Lips-Gel high-dose group had the largest number of myelinated fibers with a thicker myelin sheath at the injury site, significantly different from the free RSV group.

\section{Conclusions}

In the present study, an injectable PLGA-PEG-PLGA thermogel containing RSV liposomes was prepared. The thermogel was a sol at $30^{\circ} \mathrm{C}$ and capable of conversion into a gel at body temperature. Gels formed in situ gradually degrade in the body. Since encapsulated RSV should pass through the liposome bilayer, then diffuse from the gel, the RSV-LipsGel demonstrated stable drug release in vitro without significant initial burst compared with the RSV-Gel. In vivo pharmacological experiments indicated that the RSV-Lips-Gel promoted repair of injured sciatic nerves in rats compared with other groups. The present study demonstrated that a hybrid drug delivery system comprising liposomes and temperature-sensitive hydrogels can achieve long-lasting and stable drug release and can be administered locally to improve the pharmacological activity of the drug. Further research is required to evaluate the pharmacokinetics of such a delivery system in vivo.

\section{Data Availability}

The data used to support the findings of this study are available from the corresponding author upon request.

\section{Conflicts of Interest}

The authors declare no conflicts of interest.

\section{Authors' Contributions}

Hui Yao and Hao Lu contributed equally to this work. All authors were involved in the experiments. Yao was responsible for the design of the experiment, Lu was responsible for the statistical design, Zou and Chen implemented the experiments, and Professor $\mathrm{Xu}$ was responsible for the overall coordination of the study.

\section{Acknowledgments}

This study was sponsored by the Qingmiao Foundation of Hubei University of Traditional Chinese Medicine.

\section{References}

[1] A. Y. Mekaj, A. A. Morina, C. I. Bytyqi, Y. H. Mekaj, and S. B. Duci, "Application of topical pharmacological agents at the site of peripheral nerve injury and methods used for evaluating the success of the regenerative process," Journal of Orthopaedic Surgery and Research, vol. 9, no. 1, p. 94, 2014.

[2] R. Li, Z. Liu, Y. Pan, L. Chen, Z. Zhang, and L. Lu, "Peripheral nerve injuries treatment: a systematic review," Cell Biochemistry and Biophysics, vol. 68, no. 3, pp. 449-454, 2014. 
[3] Y. C. Lin, M. Ramadan, M. van Dyke et al., "Keratin gel filler for peripheral nerve repair in a rodent sciatic nerve injury model," Plastic and Reconstructive Surgery, vol. 129, no. 1, pp. 67-78, 2012.

[4] X. Navarro, M. Vivo, and A. Valero-Cabre, "Neural plasticity after peripheral nerve injury and regeneration," Progress in Neurobiology, vol. 82, no. 4, pp. 163-201, 2007.

[5] D. Grinsell and C. P. Keating, "Peripheral nerve reconstruction after injury: a review of clinical and experimental therapies," BioMed Research International, vol. 2014, 13 pages, 2014.

[6] A. N. Queiroz, B. A. Q. Gomes, W. M. Moraes Jr., and R. S. Borges, "A theoretical antioxidant pharmacophore for resveratrol," European Journal of Medicinal Chemistry, vol. 44, no. 4, pp. 1644-1649, 2009.

[7] S. Sánchez-Fidalgo, A. Cárdeno, I. Villegas, E. Talero, and C. A. de la Lastra, "Dietary supplementation of resveratrol attenuates chronic colonic inflammation in mice," European Journal of Pharmacology, vol. 633, no. 1-3, pp. 78-84, 2010.

[8] T. A. Zykova, F. Zhu, X. Zhai et al., "Resveratrol directly targets COX-2 to inhibit carcinogenesis," Molecular Carcinogenesis, vol. 47, no. 10, pp. 797-805, 2008.

[9] Ü. Sönmez, A. Sönmez, G. Erbil, I. Tekmen, and B. Baykara, "Neuroprotective effects of resveratrol against traumatic brain injury in immature rats," Neuroscience Letters, vol. 420, no. 2, pp. 133-137, 2007.

[10] W. Zeng, W. Zhang, F. Lu, L. Gao, and G. Gao, "Resveratrol attenuates MPP+-induced mitochondrial dysfunction and cell apoptosis via AKT/GSK-3 $\beta$ pathway in SN4741 cells," Neurosci Lett, vol. 637, pp. 50-56, 2017.

[11] H. A. Bagriyanik, N. Ersoy, C. Cetinkaya et al., "The effects of resveratrol on chronic constriction injury of sciatic nerve in rats," Neuroscience Letters, vol. 561, pp. 123-127, 2014.

[12] Y. Zhang, J. Ding, D. Sun et al., "Thermogel-mediated sustained drug delivery for in situ malignancy chemotherapy," Materials Science and Engineering: C, vol. 49, pp. 262-268, 2015.

[13] L. Yu, T. Ci, S. Zhou, W. Zeng, and J. Ding, "The thermogelling PLGA-PEG-PLGA block copolymer as a sustained release matrix of doxorubicin," Biomaterials Science, vol. 1, no. 4, p. 411, 2013.

[14] L. Sercombe, T. Veerati, F. Moheimani, S. Y. Wu, A. K. Sood, and S. Hua, "Advances and challenges of liposome assisted drug delivery," Frontiers in Pharmacology, vol. 6, p. 286, 2015.

[15] Y. Zheng, Y. Cheng, J. Chen et al., "Injectable hydrogel-microsphere construct with sequential degradation for locally synergistic chemotherapy," ACS Applied Materials \& Interfaces, vol. 9, no. 4, pp. 3487-3496, 2017.

[16] Y. Liu, X. Chen, S. Li et al., "Calcitonin-loaded thermosensitive hydrogel for long-term antiosteopenia therapy," ACS Applied Materials \& Interfaces, vol. 9, no. 28, pp. 2342823440, 2017.

[17] A. Vidyasagar, S. H. Ku, M. Kim et al., "Design and characterization of a PVLA-PEG-PVLA thermosensitive and biodegradable hydrogel," ACS Macro Letters, vol. 6, no. 10, pp. 1134-1139, 2017.

[18] G. M. El-Zaafarany, M. E. Soliman, S. Mansour et al., "A tailored thermosensitive PLGA-PEG-PLGA/emulsomes composite for enhanced oxcarbazepine brain delivery via the nasal route," Pharmaceutics, vol. 10, no. 4, p. 217, 2018.
[19] X. Guo, X. Zhu, D. Liu, Y. Gong, J. Sun, and C. Dong, “Continuous delivery of propranolol from liposomes-in-microspheres significantly inhibits infantile hemangioma growth," International Journal of Nanomedicine, vol. 12, pp. 6923-6936, 2017.

[20] Y. C. Lin, M. Y. Gao, Y. J. Wu, and Y. P. Fang, "Lipid-enveloped PLGA as a hybrid carrier for sustained delivering camptothecin in ovarian cancer," IET Nanobiotechnology, vol. 11, no. 7, pp. 797-802, 2017.

[21] P. Lundahl and Q. Yang, "Liposome chromatography: liposomes immobilized in gel beads as a stationary phase for aqueous column chromatography," Journal of Chromatography, vol. 544, no. 1-2, pp. 283-304, 1991.

[22] A. L. Weiner, S. S. Carpenter-Green, E. C. Soehngen, R. P. Lenk, and M. C. Popescu, "Liposome collagen gel matrix - a novel sustained drug delivery system," Journal of Pharmaceutical Sciences, vol. 74, no. 9, pp. 922-925, 1985.

[23] F. Gómez, R. Lorza, M. Bobadilla, and R. García, "Improving the process of adjusting the parameters of finite element models of healthy human intervertebral discs by the multiresponse surface method," Materials, vol. 10, no. 10, article 1116, 2017.

[24] M. J. Dar, F. U. Din, and G. M. Khan, "Sodium stibogluconate loaded nano-deformable liposomes for topical treatment of leishmaniasis: macrophage as a target cell," Drug Delivery, vol. 25, no. 1, pp. 1595-1606, 2018.

[25] J. Zhu, Q. Wang, H. Li et al., "Galangin-loaded, liver targeting liposomes: optimization and hepatoprotective efficacy," Journal of Drug Delivery Science and Technology, vol. 46, pp. 339-347, 2018.

[26] N. Attal, G. Filliatreau, S. Perrot, F. Jazat, L. di Giamberardino, and G. Guilbaud, "Behavioural pain-related disorders and contribution of the saphenous nerve in crush and chronic constriction injury of the rat sciatic nerve," Pain, vol. 59, no. 2, pp. 301-312, 1994.

[27] A. Fey, M. Schachner, and A. Irintchev, "A novel motion analysis approach reveals late recovery in C57BL/6 mice and deficits in NCAM-deficient mice after sciatic nerve crush," Journal of Neurotrauma, vol. 27, no. 5, pp. 815-828, 2010.

[28] B. Colzani, G. Speranza, R. Dorati et al., "Design of smart GE11-PLGA/PEG-PLGA blend nanoparticulate platforms for parenteral administration of hydrophilic macromolecular drugs: synthesis, preparation and in vitro/ex vivo characterization," International Journal of Pharmaceutics, vol. 511, no. 2, pp. 1112-1123, 2016.

[29] Z. Zhang, X. Wang, R. Zhu et al., "Synthesis and characterization of serial random and block-copolymers based on lactide and glycolide," Polymer Science Series B, vol. 58, no. 6, pp. 720-729, 2016.

[30] W. Zhen, Y. Zhu, W. Wang, and Z. Hou, "Synthesis and properties of amphipathic poly(D,L-lactide-co-glycolide)polyethylene glycol-poly(D,L-lactide-co-glycolide) triblock copolymers," Australian Journal of Chemistry, vol. 68, no. 10, article 1593, 2015.

[31] S. Bashir, Y. Y. Teo, S. Ramesh, and K. Ramesh, "Physicochemical characterization of $\mathrm{pH}$-sensitive $\mathrm{N}$-Succinyl chitosan- g -poly (acrylamide- co -acrylic acid) hydrogels and in vitro drug release studies," Polymer Degradation and Stability, vol. 139, pp. 38-54, 2017.

[32] S. Zhang, X. Meng, Z. Wang et al., "Engineering hot-melt extruded solid dispersion for controlled release of hydrophilic drugs," European Journal of Pharmaceutical Sciences, vol. 100, pp. 109-115, 2017. 
[33] M. J. G. Bradman, F. Ferrini, C. Salio, and A. Merighi, "Practical mechanical threshold estimation in rodents using von Frey hairs/Semmes-Weinstein monofilaments: towards a rational method," Journal of Neuroscience Methods, vol. 255, pp. 92103, 2015.

[34] D. C. Hammell, L. P. Zhang, F. Ma et al., "Transdermal cannabidiol reduces inflammation and pain-related behaviours in a rat model of arthritis," European Journal of Pain, vol. 20, no. 6, pp. 936-948, 2016. 